\title{
A stellar coronograph for the COME-ON-PLUS adaptive optics system ${ }^{\star}$
}

\section{Description and performance}

\author{
J.-L. Beuzit ${ }^{1,2}$, D. Mouillet ${ }^{1}$, A.-M. Lagrange ${ }^{1}$, and J. Paufique ${ }^{1}$ \\ 1 Laboratoire d'Astrophysique de l'Observatoire de Grenoble, UMR 5571, Université J. Fourier, BP. 53, F-38041 Grenoble \\ Cedex 9, France \\ 2 DESPA, Observatoire de Paris, URA 264, Place J. Janssen, F-92195 Meudon Cedex, France
}

Received July 26, 1995; accepted December 19, 1996

\begin{abstract}
The detection of faint emissions very close to much brighter objects is required in several different fields of astrophysics, for example in the search for planetary disks. We present here a coronograph that we built to work with COME-ON-PLUS, the ESO adaptive optics system, high angular resolution being of key importance in allowing observations close to a central, occulted object. The first results are presented and analyzed in order to establish the performance of this instrument in terms of contrast and detectability of faint emissions close to a central star.
\end{abstract}

Key words: atmospheric effects - methods: data analysis — instrumentation: miscellaneous — stars: circumstellar matter

\section{Introduction}

The difficulty of observing faint emissions in the close environment of bright objects, like stars, increases with the contrast. Yet, many fields of astrophysical research depend on this ability. One important field is the study of the close environment of IRAS excess stars where the aim is to detect the origin of such infrared (IR) emissions as either disks, or red and faint objects. Alternatively, very faint structures around a number of Herbig stars could be imaged.

For instance, a circumstellar disk has been imaged around $\beta$ Pictoris (Smith \& Terrile 1984), using a coronograph with a $7^{\prime \prime}$ diameter occulting mask and a Lyot

Send offprint requests to: J.-L. Beuzit

* Based on observations collected at the European Southern Observatory, La Silla, Chile. mask on a 2 meter class, classical (i.e. without adaptive optics) telescope. This first detection has been followed by observations of this disk closer to the star, using two different approaches. A first one consisted of using image tip-tilt correction before the coronograph (Golimovski et al. 1993), and allowed the detection of the disk down to $2^{\prime \prime}$ from the star, and quantitative measurements to $2.5^{\prime \prime}$, to be compared to $6^{\prime \prime}$ obtained without correction. The second method used an anti-blooming detector to achieve a very high dynamic range instead of using a coronograph (Lecavelier et al. 1993) and enabled a similar performance to be reached. The scientific interest in the vicinity of the star is important: the physical nature of the medium is supposed to change within $2^{\prime \prime}$ and this coincides also with the expected location of planets and their area of influence. The thermal emission of the dust has also been imaged at $10 \mu \mathrm{m}$ (Lagage \& Pantin 1994). This provides complementary physical information.

A systematic search for similar disks around other main sequence (MS) stars has also been performed (Smith et al. 1992). No other disk was found among approximately 100 candidates. The reasons for these non detections are probably a combination of three facts (Lagrange 1994). The disks are possibly intrinsically fainter than that of $\beta$ Pictoris (according to IRAS indications), the disks may be unfavourably oriented, and they may also be closer to the star.

In the context of such studies, scientific results and constraints would benefit greatly if one could observe faint (typically less than 15 magnitude per $\operatorname{arcsec}^{2}$ ) and extended structure much closer (typically closer than $2^{\prime \prime}$ ) to the bright central object. Such performance requires the highest possible angular resolution as well as the use of techniques which provide a very high dynamic range. 
Theoretical calculations indicate that the quality of coronographic images depends critically on the angular resolution. The improvement with the use of partial image correction has already been demonstrated by Malbet et al. (1992) on the COME-ON adaptive optics system and by Golimovski et al. (1993) with their Adaptive Optics Coronagraph. On the basis of the COME-ON experiment, we have built a coronograph to work with the COME-ON-PLUS adaptive optics system on the ESO 3.6 meter telescope at La Silla, which provides diffractionlimited images in $K$ band $(2.2 \mu \mathrm{m})$, i.e. a resolution of $0.12^{\prime \prime}$. It was hoped to be able to use masks smaller in diameter than $1^{\prime \prime}$, which is significantly smaller than the ones used without or with partial image correction (respectively, typically $4^{\prime \prime}$ and $2^{\prime \prime}$ ). In 1994, six nights were devoted to testing the coronograph and measuring the performance of the $\mathrm{AO}+$ coronograph system in terms of dynamic range and the ability to observe faint emissions close to a central, bright object.

In Sect. 2, we review the theoretical reasons that support the use of a coronograph with adaptive optics. We describe the coronograph in Sect. 3 and we present in Sect. 4 a detailed description of the reduction procedure for this particular type of data. The resulting capabilities of the whole procedure are presented and the different sources of uncertainty are evaluated in Sect. 5. These are critically compared to theoretical calculations and the performance of other existing systems in Sect. 6. Conclusions and perspectives to this instrumental work are given in Sect. 7 .

\section{Need for an adaptive optics coronograph}

\subsection{Adaptive optics}

The angular resolution of ground-based telescopes is usually not limited by the quality of the telescope optics, but rather by the atmospheric turbulence. This phenomenon, called seeing, is due to random temperature fluctuations in the atmospheric layers which induce local variations of the index of refraction resulting in distortions of the incoming wavefront (Roddier 1981). Regardless of the telescope aperture, the ultimate resolution is rarely better than $0.5^{\prime \prime}$ even in good astronomical sites. Another effect of the seeing is to degrade the imaging sensitivity, by spreading the collected photons over a much larger area. A posteriori techniques have been developed to overcome the effects of the atmospheric turbulence on the image quality, using digital post-processing: speckle interferometry, deconvolution from wavefront sensing, etc. Nevertheless a more promising approach is to compensate in real-time the atmospherically induced wavefront distortion by an adaptive optics (AO) system. In this technique the light coming from the object under study, or from a nearby reference star, is analyzed by a wavefront sensor. Using this information, the surface of a deformable mirror is modified in real-time by a servo control system. The resulting wavefront is nearly planar and the resolution becomes close to the diffraction limit of the telescope (Alloin \& Mariotti 1994).

\subsection{Coronography}

Collecting information close to a star implies the use of small masks. Theoretical calculations enable the expected performance of coronographic techniques to be simulated (Malbet et al. 1996), and the influence of the respective shapes of the occulting mask and of the Lyot stop to be explored. Basically, the critical parameter is the relative size of the occulting mask to the point source response extension. It strongly affects the rejection capability of the system, as well as the efficiency of the Lyot stop. For instance, we have calculated that the use of a $0.8^{\prime \prime}$ occulting mask leads to simulated rejection rates of 335 in the case of a perfectly flat wavefront, 215 for AO corrected images, but only 50 for tip-tilt corrected images, and 5 in case of no correction. In other words, to obtain the same rejection rate as the $\mathrm{AO}$ corrected images would require the use of a mask that is two times larger with tip-tilt corrected images, and 3.5 times larger without correction. This particular numerical example (Fig. 1) is calculated for the case of $1^{\prime \prime}$ seeing and with a 3.6 meter telescope at $2.2 \mu \mathrm{m}$.

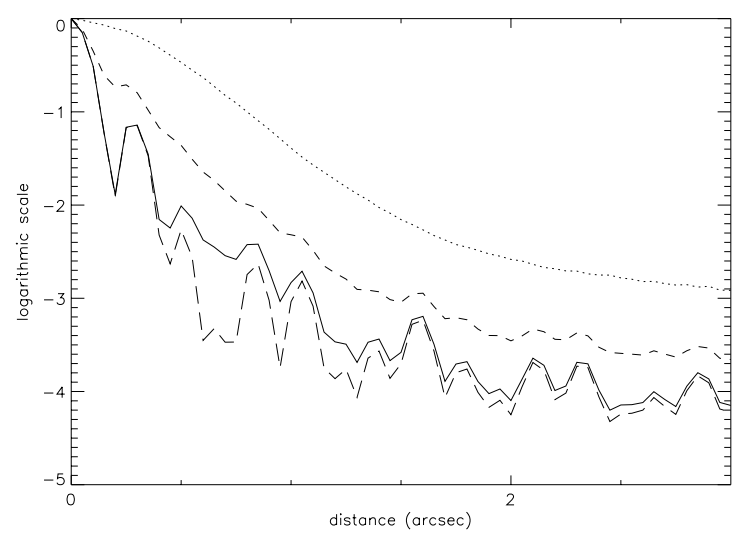

Fig. 1. Simulated profiles of PSF obtained with a 3.6 meter telescope at $2.2 \mu \mathrm{m}$ under atmospheric Kolmogorov turbulence conditions (seeing $=1^{\prime \prime}$ ) and different correction modes. The full line indicates the profile that the adaptive optics system should generate on a bright star, compared to the profile in a perfect case of flat wavefront (long-dashed line) and the case of the tip-tilt correction (dashed line) and no correction at all (dotted line). Note that the discrepancy between the calculated feature for the perfectly flat wavefront and the Airy pattern only comes from the numerical calculation, and has no link with atmospheric turbulence 


\section{Description of the COME-ON-PLUS coronograph}

\subsection{The COME-ON-PLUS adaptive optics system}

The COME-ON-PLUS instrument (Gendron et al. 1991), developed in collaboration by ESO and several French institutes and companies (Observatoire de Paris, ONERA, Laserdot and LEP), routinely achieves diffraction-limited images at near infrared wavelengths (down to $1.65 \mu \mathrm{m}$ ) on the ESO 3.6 meter telescope at La Silla (Rousset et al. 1994; Beuzit 1995). The images are recorded on the $256 \times$ 256 NICMOS 3 detector of the SHARP II infrared camera (Hofmann et al. 1992) using an image scale of $0.05^{\prime \prime} /$ pixel, which leads to a total field of view of $12.8^{\prime \prime} \times 12.8^{\prime \prime}$. The camera features the standard $J, H, K$ and short $K$ broadband photometric filters.

For reference star magnitude lower than 10, i.e. at high photon flux, the best FWHM is around $0.1^{\prime \prime}$ and is obtained in the $H$ and $J$ bands. In the $K$ band, the $0.12^{\prime \prime}$ angular resolution and a typical Strehl ratio, i.e. the flux concentration with respect to the perfect image case, of 30 to $40 \%$ are achieved.

\subsection{The coronographic mode}

The optical sketch of the COME-ON-PLUS coronograph is shown in Fig. 2. Unlike most of the existing coronographs, it is composed exclusively of reflecting surfaces to avoid chromatic effects when switching from one wavelength to another. The occulting mask, a flat metallic mirror with a calibrated central hole, is located at the F/45 output focus of the adaptive optics bench (Mc2). The star light goes through the hole while the image of the surrounding region is reflected to the camera. The Mc3 spherical mirror collimates the light beam onto the Lyot stop (Mc4). The second spherical mirror (Mc5) images the focal plane onto the infrared detector with the same $\mathrm{F} / 45$ aperture. Additional flat mirrors are used to allow a fast switching from the coronographic mode to the standard one without removing the coronograph itself.

Different occulting masks are available with sizes ranging from $245 \mu \mathrm{m}$ to $1.6 \mathrm{~mm}$ i.e. projected diameters from $0.3^{\prime \prime}$ to $2^{\prime \prime}$, the image scale of the ESO 3.6 meter telescope being $790 \mu \mathrm{m} \mathrm{arcsec}{ }^{-1}$ at the $\mathrm{F} / 45$ focus. The masks were made from stainless steel disks in which holes were first drilled. They were then optically polished at a surface quality of $\lambda / 4$ at $0.55 \mu \mathrm{m}$ and silver coated.

The Lyot stop or apodizing mask, located at a telescope pupil image, is used to block the diffracted light from the edge of the telescope's primary and secondary mirrors and from the secondary support (including the spider arms). In doing so, it needs to partially obstruct the pupil image. Two masks, obtained by a microphotolithographic process, were produced by the LARCA (LAboratoire de Réalisation de Composants pour l'Astronomie) at the Observatoire de Paris. They correspond to relative ob-

\section{Data reduction}

\subsection{Description of the data}

The observing procedure for a target star consists in recording exposures of that target with the occulting mask as well as exposures made without the mask to measure the point spread function (PSF). The same information for a comparison star is necessary in the reduction procedure as described in Sect. 4.3. Usually two comparison stars are observed for cross confirmation purposes, under the same conditions, with and without the mask. For each objects, the sky emission is measured at a distance of, typically, $30^{\prime \prime}$ away from the target object. Individual exposure times are constrained by the dynamical range of the detector. With the $256 \times 256$ SHARP II near IR camera on bright targets (mag $=3-6)$, they are typically a few seconds. Data cubes of typically 100 images are recorded. In addition flat-field and dark exposures are recorded at the beginning and the end of the night.

\subsection{Reduction of instrumental effects}

\subsubsection{Bad pixels removal}

What is referred to here as "bad pixels" are pixels which behave abnormally in comparison to the whole detector. These include pixels with a non-linear response, dead pixels and pixels with excessive noise or characteristics that vary from one night to another. They are easily detected by their strong deviation from the mean local intensities. Their value is replaced by the median value of the neighbouring pixels. This procedure is necessary for a few hundred pixels in the $256 \times 256$ image, and has no deteriorating effect on the angular resolution.

\subsubsection{Dark exposures}

With the detector used the dark signal is very stable and reproducible, but depends strongly on the exposure time (especially for short exposures). Dark exposures of equal duration as the exposure on the astronomical targets are recorded.

\subsubsection{Sky and flat field}

The sky emission is measured $30^{\prime \prime}$ away from the star. The implicit assumption is that no significant variation occurs on this scale. Note that to take into account this variation to a first order would imply additional observations of the sky on the opposite side of the object, but doing this would also increase the delay between the object and the reference exposures. The sky subtraction also removes the instrumental background. 

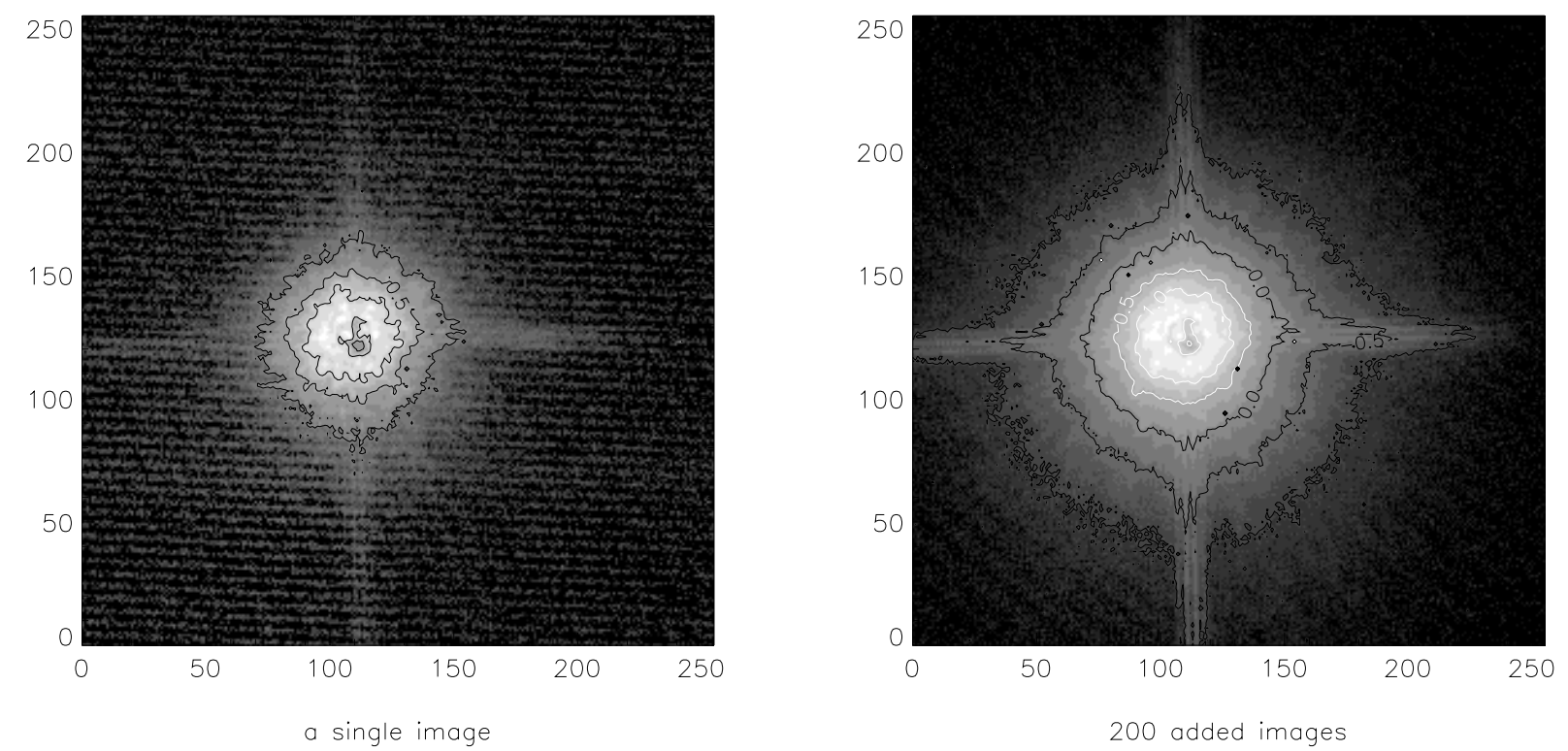

Fig. 3. Residual light from a star behind the mask, for a single exposure a) and 200 added images b). The scale is logarithmic: the inhomogeneities in the wings are at very low intensities compared to the peak of the star

change, which never happened in our observations. Then all images are simply added.

\subsection{Correction with a comparison star}

After the mask, most of the stellar light is removed. However, the wings of the PSF are still present outside the occulting mask and are much stronger than the circumstellar emissions that we wish to image. We may be able to remove the residual light from the central object, since its shape is expected to exclusively depend on optics and correction. This step is critical and some difficulties are generally encountered.

A possible method is to use the star itself to estimate the diffracted light profile on a region of the image where no circumstellar emission is expected. The profile is then azimuthally extended to the whole image, following Golimovski et al. (1993) in the observation of the $\beta$ Pictoris disk. Yet the definition of the area used to evaluate the light to be subtracted is critical. Indeed, it involves some a priori knowledge of the observed circumstellar emission. In particular, in case of extended emission it seriously affects the shape of detected non central emission, especially close to the star. In order to avoid such possible biases, which are difficult to estimate, we prefer to use a comparison star observed under the same conditions. This comparison star is chosen to be angularly close to the target and of similar magnitude and stellar type in order to get the same adaptive optics correction. For the same reason, it is observed very close in time as the target object. We also take care to avoid any reference star suspected to have a companion or some circumstellar material. The reduction procedure of this comparison is exactly the same as for the main target. The residual light from the central object is then subtracted, after proper scaling to the brightness of the target, by a multiplicative factor. After this procedure, only non-central emission should remain.

The reasons why this procedure is delicate are the following: First, the gradients in the PSF feature we subtract are steep (radial dependence in $r^{-3}$ ) so that any slight difference in the shape of the residual light from the star affects the efficiency of the procedure. A possible offset that may have occurred between the observations of the target and the comparison induces in the signal after subtraction a strong deviation from the zero in the region close to the mask with a highly positive area on one side opposite to an highly negative one. We correct this effect by recentering down to a precision of $1 / 8$ pixel. Exploring higher precision do not clearly improve the result and the remaining deviation from zero after the subtraction of the central light comes from the following effects more than from the centering precision. Second, due to the photon noise and also to the fact that very high modes of the image correction are imperfect, some azimuthal inhomogeneities remain and vary from one single exposure to another. They are reduced by increasing the number of exposures, but the finite total exposure time still causes uncertainties to the evaluation on the shape of the residual light as can be seen in Fig. 3. This uncertainty, coupled with the photon noise, is quantified by the measure of the signal variance along a data cube, represented as a function of the distance from the star in Fig. 5 (long dashed line curve). Third, the global quality of the image correction varies on time-scales which depend on weather conditions (minutes to hours), 
and on the star intensity and position in the sky. Such a slight variation implies that both profiles may be not exactly similar anymore. This clearly appears when one divides the image of the object by the comparison one. The result sometimes show a dependence on the distance from the star. This occurs when the two stars are observed more than one hour apart. With shorter delays, the division is flat over most of the field of view. We can then derive a constant scaling factor. The dominant source of uncertainty is no longer due to this determination.

Finally, after all these corrections, we are able to quantify the resulting uncertainty in two ways. The first one is to measure the residual light in the reduced image (star corrected by the comparison) where no emission is seen. The second one is to apply the same method to two comparison stars observed respectively before and after the target.

\section{Performances of the coronograph}

\subsection{Efficiency of the coronograph on a single object}

The efficiency of the coronograph can be measured by comparing images of the same object with and without the occulting mask (Fig. 4). As exposure times are much shorter in the second case, the profile is normalized to one second exposure. The coronograph parameters are a $0.8^{\prime \prime}$ occulting mask and a Lyot stop occulting $10 \%$ of the light in the following pupil plane.

The most spectacular effect is the gain in terms of dynamic range. The rejection rate, i.e. the ratio of the brightest pixel of the respective images, is larger than 100. As a direct consequence, it becomes possible to integrate longer in order to get a good signal-to-noise ratio $(\mathrm{S} / \mathrm{N})$ on the wings of these profiles. This provides the ability to detect fainter emissions. We have to stress the fact that the reduction procedure described above assumes that no part of the detector is saturated and that every pixel operates in the linear regime.

A second effect of the coronograph is the lowering, by a factor of 2 , of the outer profile of the occulted star outside the area of efficiency of the occulting mask. This efficiency of the Lyot stop comes from the fact that the occulting mask rejects most of the light of the occulted object in the border of the next pupil. As the delicate step of the reduction procedure is the removal of this profile wings (Sect. 4.3), this gain, specific to coronographic techniques, is valuable.

\subsection{Efficiency of the whole procedure}

As already seen in Sect. 4, the remaining light from the central object is removed by the comparison with another
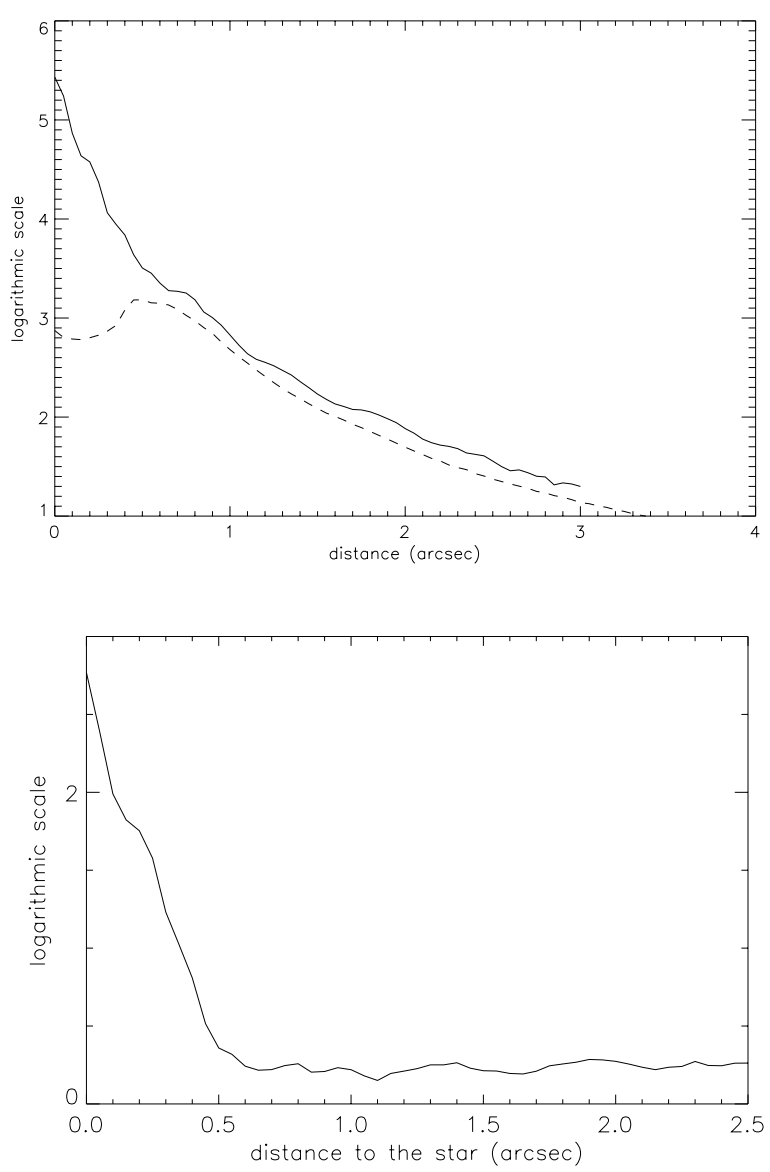

Fig. 4. Respective normalized profiles of a star without the occulting mask (full line) and with the mask (dashed): (top). Horizontal axis is scaled in arcsec. The ratio of these two profiles is plotted (bottom) on a logarithmic scale

star. We estimate the noise of the whole procedure by applying it to two point-like sources, or on the part of the resulting image where no emission is expected. The deviation from zero indicates the faintest detectable emission around a given occulted object. Figure 5 presents the radial dependence of this limit. With such observational parameters the final correction by the reference object leads to a detectable limit around 20 times fainter than the diffracted light of the central object at $2^{\prime \prime}$. In terms of dynamic range, a gain of 100000 further than $2^{\prime \prime}$, or 40000 at $1.5^{\prime \prime}$, is obtained if one compares the peak intensity of the non occulted star with the residuals after the whole procedure.

\subsection{Effect of observational parameters on the performance}

The derived detection limit obviously depends on the particular instrumental (mask sizes, filters) and observational parameters (brightness of star, airmass, weather 


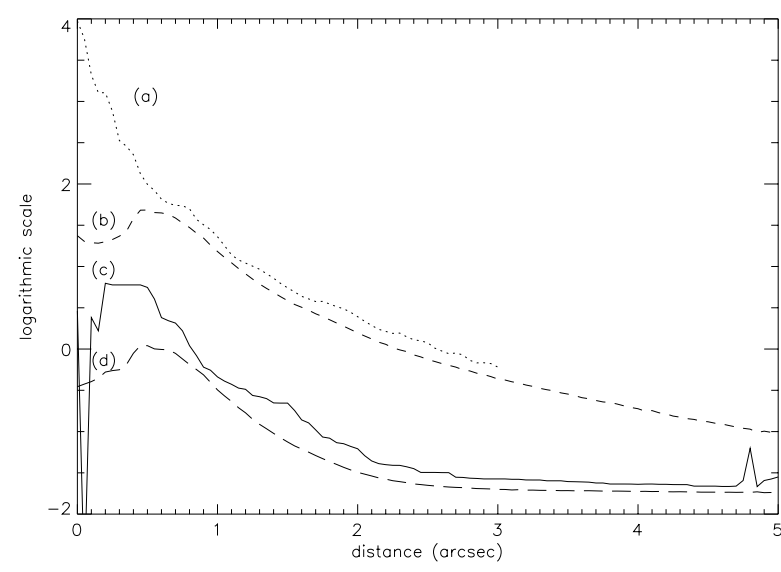

Fig. 5. Profile, on a logarithmic scale, of the rms residual light after the whole reduction procedure as a function of the distance from the star c). This indicates the detection limit in the present case which, at $2^{\prime \prime}$, is $10^{5}$ fainter than the peak intensity of the star observed without the mask with the same angular resolution a). The difference between the detection limit and the profile of the occulted star b) shows the efficiency of the reference profile subtraction. Profile $\mathbf{d}$ ) is the rms variation of individual images from the average image in a cube (for the reference and the object). This illustrates the cumulative effects of the photon noise and the high turbulence modes. The discrepancy between the observed limit level c) and the expected one $\mathbf{d}$ ), due to variations along a single cube, is due to small variation between the two object observations. Further than $2^{\prime \prime}$, the detection limit level is given by the precision on the sky and is related to the total exposure time

conditions and variability, exposure times..). Therefore, they should be considered as illustrative rather than as upper limits to what can be achieved. One may basically extrapolate corresponding limits for observations under different conditions, or use them to fit the instrumental possibilities to specific scientific requirements.

In a data cube, the uncertainty of the measurement is derived from the rms variations of the individual images compared to the average image (Fig. 5d). The precision of the average image increases as the square root of the number of images. In a cube of dark and flat field corrected data, the uncertainty of the signal has various origins, depending on the distance from the star. Further than $2^{\prime \prime}$ from the star, in low flux area, the detector read out noise dominates. It is then consistent with the uncertainty of the dark signal and the sky emission (for typical exposure times of seconds or less). Closer to the star, as the flux gets larger, the $\mathrm{S} / \mathrm{N}$ improves but the absolute uncertainty per pixel also increases, and gets larger than the read out noise. Major sources of uncertainties here are the photon counting noise and also the irregularities on the wings of the PSF due to the imperfectly corrected high order and fast modes of the turbulence. As a consequence, in the complete field of view, the precision of the measurement of the residual light may be directly enhanced by increasing the total exposure time.

The precision of the whole procedure (Fig. 5b), i.e. after subtraction of the reference signal, may not be better than the one of the target and the reference. This level is reached in read out noise dominated regions, i.e. further than $2^{\prime \prime}$. This means that, close to the star, the critical step of the procedure is the subtraction. We already mentioned that this may come from a globally different behaviour of the correction for both stars because of small differences of brightnesses, position in sky, observation time, and numerical subtraction (multiplicative factor determination, offset). To investigate the very close environment of stars, one needs a long total exposure time and an exact similarity between the reference and target stars. They should also be angularly very close and observed less than one hour apart, or even less when the turbulence changes rapidly. The observing strategy should then aim to optimize the compromise between a large total exposure time and short delays between the various exposures. This depends on the distance and magnitude of the investigated emissions.

Other important parameters are the occulting and Lyot masks sizes. One needs to use small occulting masks to investigate the very close vicinity to the star, even though this leads to lower rejecting capabilities of the occulting and Lyot masks (see for instance Sect. 2; Malbet et al. 1996) and smaller exposure times.

One comment on the final angular resolution: This is critical in the whole reduction procedure as it allows the use of small masks, whether or not the observer is interested in achieving angular resolution. If the highest possible angular resolution is not the observational priority, it is always possible, a posteriori, to degrade the resolution. The limit detection, in terms of flux per element resolution, correspondingly increases.

\section{Comparison with expected performance and other systems}

Section 5 gives the effective performance of the instrumental configuration used. We discuss them in comparison with theoretical calculations and others systems.

First, the rejection capability of the coronograph appears to be in good agreement with theoretical expectations, even though it is slightly reduced. The following effects may explain the remaining discrepancy. First, the optics are not perfect. In particular, the mirror scattering decreases the efficiency of a coronograph. For instance, the latest observations occurred after the primary mirror had been re-aluminized and the improvement in the image quality was spectacular. Second, the wavefront 
after the adaptive optics system is not perfectly flat and the wings of the PSF are correspondingly modified. Also, since the efficiency of the coronograph critically depends on the accurate centering of the star, a slight variation in the position during observation, possibly due to a small mechanical deformation, affects the whole shape of the residual light profile.

The importance of the star position stability underlined below explains why observing with little, or no image correction does not allow the use of very small masks. Consequently, the close vicinity of the star may not be investigated without AO. Quantitatively, no precise measurements have been achieved closer than $3^{\prime \prime}$ from a star without AO correction (Kalas \& Jewitt 1995) or closer than 2.5" with tip-tilt correction (Golimovski et al. 1993). This is to be compared to our present limit of $1.5^{\prime \prime}$, where the uncertainty of the whole observational and reduction procedure discussed in Sect. 5.2 is lower than the disk brightness (Mouillet et al. 1995, here after Paper II). We have to stress that our observational parameters were optimized for observation close to the star. Different observational parameters would be chosen to observe at greater distances. A larger mask would provide a better $\mathrm{S} / \mathrm{N}$ with longer exposure times. The compromise between the pixel sampling and the field of view depends on the particular application concerned.

Finally, the use of a coronograph may also be discussed. In terms of the requested dynamic range, our detection limit at $2^{\prime \prime}$ and beyond is around 100000 times fainter than the peak intensity of the non occulted star observed with the adaptive optics system under the same conditions. This limit could even be improved by a total exposure time larger than 10 minutes since the limiting factor is in this case the low flux noise. Yet to achieve the same result without a coronograph, the detector intrinsic dynamic range (in the linear regime) would need to be much larger. Indeed, the subtraction of a reference profile requires a very good signal to noise ratio in the wings and this implies a significant number of electrons in the low flux regions.

\section{Conclusion and perspectives}

Adaptive optics enables the use of coronographs with very small mask sizes. We have described a stellar coronograph that we built to work with the ESO COME-ON-PLUS adaptive optics system and we have given its performance in terms of dynamic range and detection capabilities. The detection limit for faint emissions have been discussed and quantified as a function of the distance from the central star. The critical step of the reduction procedure is the remove of the diffracted light from the occulted star. It is very sensitive to the quality of the adaptive optics correction, in terms of width of the PSF but also of regular- ity and stability of the wings. On this basis, longer total exposure times will enhance the resulting performance; and finally the use of apodizing masks are under study to improve rejection capability of the coronograph. The achieved performance of the present configuration is unprecedented in terms of angular resolution over the whole image and in the vicinity of the star. Both characteristics are critical in the observational study of various astrophysical objects. The first astronomical results will be described in Paper II.

Acknowledgements. We are grateful to all colleagues involved in the development of the adaptive optics coronographic mode, especially P. Kern, C. Marlot, V. Serpette and S. Wang. We also wish to thank N. Hubin, P. Léna, A. Vidal-Madjar, F. Malbet and J.-M. Mariotti for helpful discussions and M. Cullum for comments on the manuscript. Additional thanks are extended to the COME-ON-PLUS team as well as the ESO La Silla staff, especially M. Maugis, E. Prieto, P. Le Saux for their active help during the integration and tests of the coronograph on the 3.6 meter telescope.

\section{References}

Alloin D., Mariotti J.-M., 1994, Adaptive Optics for Astronomy, NATO ASI Series C. Kluwer Academic Publishers

Beuzit J.-L., 1995, PhD Thesis

Clarke C.J., Pringle J.E., 1991, MNRAS 249, 588

Gendron E., Cuby J.-G., Rigaut F., et al., 1991, Active and Adaptive Optics, Ealey M.A. (ed.) Proc. SPIE 1542, 297

Golimowsky D.A., Durrance S.T., Clampin M., 1993, ApJ 411, 41

Hofmann R., Blietz M., Duhoux P., et al., 1992., Progress in Telescope and Instrumentation Technologies, Ulrich M.-H. (ed.) Proc. ESO 42, 687

Kalas P., Jewitt D., 1996, AJ 111, 1347

Lagage, P.O., Pantin, E., 1994, Nat 369, 628

Lagrange A.-M., 1994, ESO Messenger 76, 23

Lecavelier A., Perrin G., Ferlet R., et al., 1993, A\&A 274, 877

Malbet F., 1992, PhD Thesis

Malbet F., 1996, A\&AS 115, 161

Mouillet D., Lagrange A.-M., Beuzit J.-L., Renaud N., 1995, A\&A (in press)

Roddier F., 1981, Progress in Optics, Wolf E. (ed.) XIX, North Holland

Rousset G., Beuzit J.-L., Hubin N., et al., 1994, Adaptive Optics in Astronomy. In. Ealey M.A. \& Merkle F. (eds.) Proc. SPIE 2201, 1088

Smith B.A., Fountain J.W., Terrile R.J., 1992, A\&A 261, 499

Smith B.A., Terrile R.J., 1984, Sci 226, 1421 